\title{
Designing Service Environments for Virtual Scene Applications
}

\author{
John Gammack \\ Institute for Integrated and Intelligent Systems, Griffith University, Queensland, Australia 4131 \\ j.gammack@griffith.edu.au
}

\begin{abstract}
Continuing internet ubiquity enables new kinds of applications previously restricted to powerful platforms unavailable to home consumers. These applications are characterized by extensive interactivity, by high quality multimedia presentation, and by real-time qualities. Typical emerging application areas include entertainment, and cybertourism. Achieving this requires integration between technologies and the end user's information needs and expectations. Virtual reality, cluster and grid-computing technologies provide a suitable basis for applications that operate in the timeframes consumers expect. The design of services and applications will require an understanding of the dynamic forms of intelligent user behaviour and interactivity involved. This paper draws from a case study to suggest theory relevant to design of an integrated environment, and is illustrated with cybertourism oriented applications.
\end{abstract}

\section{Introduction}

As the home penetration and functionality of the Internet continues, traditional human activities take new forms. Business has already reconfigured its activities around the Internet, and consumers are also increasingly doing so. The Internet currently provides not only an open and general platform with considerable computing potential, but also provides information and services over familiar web interfaces. As this integrates with emerging consumer-oriented devices this gives a basis for new services and multimedia applications taking advantage of the ubiquity and power of the underlying technology. In turn, these applications will require higher order knowledge of the media objects and their meaning in the consumer's world as well as the specific architectures that can ensure effective service delivery.

Such applications can be expected to initially operate over the Internet, and will eventually run on consumer-oriented target devices. These applications will entail suitable facilities at the server end, and require high performance capabilities to enable the interactivity and the presentational requirements to operate in acceptable timescales. Quality-of-service will be critical for acceptance, and these requirements will generate both technical and business issues.

In this paper we focus on relevant consumer aspects more than the technical, although theory would require integrating both. The dependability, load and speed requirements for consumer applications will entail a grid or cluster approach, and the pricing, delivery, level of service and mechanisms for on-demand, interactive multimedia will require business and consumer decision inputs.

\section{Literature review}

E-business, and e-service are driving high performance computing. Tourism, in particular is information intensive, and a driver of economic development. For example virtual tours: preimagining an experience predisposes it to happen. Affordable alternatives to physical tourism, widening access and accessibility have considerable future potential. Environmental and heritage management and preservation is also enabled allowing immersive experiences and interaction without damaging the resource. [1,2]. This assists in preservation of heritage sites and web distributed to basicspecification machines.

More complex engagements can occur with cities or other sites with arbitrarily detailed navigation. Here consumer choices determine presentation dynamics, and fast responsiveness to these is required. With semantics, customized tourism systems can take user preferences into account. [3]. A sense of orientation, presence, customized service and experience is gained, all valuable in retaining consumer involvement. Computationally intensive server processing however, is required for effectiveness of such applications, which may be presented in the client's browser using today's free VRML plug-ins and in future the extensible 3d (X3d) standards developed through the Web 3D consortium at http://www.web3d.org/x3d/.

\section{Case Study}

"Beachtown" is an Australian heritage city with excellent tourism facilities. A "virtual Beachtown" allows prospective visitors to construct visitation 
scenarios to arbitrary levels of detail. Other applications predicated on the virtual world include planning and heritage information and environmental impact of proposals.

We built a VRML model of Beachtown and surveyed a panel of users following increasingly computationally intensive levels of engagement. This showed that interactive multimedia was acceptable for information discovery but that a VRML experience increased likelihood of visitation. [4]. Rendered animations were disliked, and the strong view was panoramic photographs would be better.

Our general finding was that more complex information environments increase attention and involvement, increase memorability, willingness to purchase online and also the desire to compare the picture with the reality.

This work motivates more immersive experiences beyond simple renderings and basic processing capability, and requires associated theory.

\section{Implications for future research}

Supporting real time, photogrammetric quality imaging goes beyond single processor capability. In addition, slow pre-loading of scenes and active reconstruction as viewers change direction or focus on details, (such as café menu prices) which can change in real time imply a need for integration with powerful processing capability. Emergent navigation methods are signalled as the web evolves towards more user designed interactivity. As the activities of users cannot be anticipated in advance, nor the details to which they might attend, a design environment allowing for user-centred reconstruction is required.

A "late binding" philosophy of design environments recognises that users operate in dynamic contexts, which bring active meaningbearing structures to their application use decisions. End-user developed systems have higher satisfaction and individual utility, but can lack quality and sophistication. But third party developments lack user context, freezing requirements inappropriately. If end-users dynamically construct their own patterns of interaction, a generic environment allowing rapid, tailored generation is required to allow application consumers, not system developers, to "design the interaction". This will require dynamic scene construction using metadata user communities understand and indicate in their navigation models.

A cybertourism application in "consumer realtime" is representative. The BSP model [5] provides a framework and programming library for parallel software allowing software portability over different underlying architectures, and capable of reading VRML files, allowing reuse. OpenGL Performer can use a cluster facility and visualization for interactivity. Tagging consumer events enables semantic patterns to be identified. Quality of service is a key feature of applications that drive grid computing.

Real-time but not safety-critical features are important in this, including "consumer real-time" where emphasis is on engagement with attention and decision-making, and acceptable response speed and smoothness applies. Cityscape modelling for virtual tours can display cinematic imaging and advertising displays (Hong Kong's infrastructure gallery exhibitions already renders cityscape views to image and visualise the harbour developments). This avoids the risk of showing rain or fog via a webcam.

In participatory environmental management, interaction is needed for establishing engagement and a sense of presence. Interactive capabilities will also promote (a) user exploration of design, planning or management options and (b) studies of humanenvironment interaction for management purposes or construction of agent-based models of process in both the tourism and planning contexts. The implicit semantic structures and public knowledge of a place is also captured in VR navigations and this will be important for designing the experience of a place allowing tourist itineraries, signposting and guidance to be designed. The possibilities for future systems by exploring these ideas are very exciting.

\section{References}

[1] T. Buehrer, L..Zhang, A. Gruen, C. Fraser, and H. Rüther, Photogrammetric reconstruction and 3D visualisation of Bet Giorgis, a rock hewn church in Ethiopia. Proceedings International CIPA Symposium

"Surveying and Documentation of Historic Sites", Potsdam September 2001 pp $18-21$.

[2] A. Gruen, F. Remondino, L. Zhang. Photogrammetric Reconstruction of the Great Buddha of Bamiyan, Afghanistan The Photogrammetric Record 19(107): 2004 pp177-199

[3] J. Fink J and A. Kobsa User modeling for personalized city tours Artificial Intelligence Review 18 2002. pp33-74

[4] J.G. Gammack and C. Hodkinson Virtual reality, involvement and the consumer interface Journal of End User Computing, 15, 4, October-December, 2003 pp80-98

[5] L.G. Valiant, (1990) A Bridging Model for Parallel Computation Communications of the ACM; New York 103-111. 